\title{
MULTIVARIATE PADÉ APPROXIMANTS FOR FINITE ELEMENT SOLUTIONS WITH COMPLEX PARAMETRIC DEPENDENCE
}

\author{
Romain Rumpler $^{1}$, Raúl Rodríguez Sánchez ${ }^{2}$, Peter Göransson ${ }^{1}$ \\ ${ }^{1}$ MWL/Centre for ECO2 Vehicle Design, Department of Aeronautical and Vehicle Engineering, \\ KTH Royal Institute of Technology, Stockholm, Sweden \\ e-mail: rumpler@kth.se, pege@kth.se \\ ${ }^{2}$ Chair of Structural Mechanics, Technical University of Munich, Arcisstraße, \\ Munich, 80333, Germany \\ e-mail: raul.rodriguez@tum.de
}

Keywords: Reduced Order Model, Padé Approximants, Fast Frequency Sweeps, Finite Element Method.

\begin{abstract}
Most engineering applications involving solutions by numerical methods are dependent on several parameters, whose impact on the solution may significantly vary from one to the other. At times an evaluation of these multivariate solutions may be required at the expense of a prohibitively high computational cost. In the present work, a multivariate finite element approach is proposed, allowing for a fast evaluation of parametric responses. It is based on the construction of a reduced basis spanning a subspace able to capture rough variations of the response. The method consists in an extension of the Well-Conditioned Asymptotic Waveform Evaluation (WCAWE) to multivariate problems, by an appropriate choice of derivative sequences, and a selection of the most relevant basis components. It is validated and demonstrated for its potential on a semi-industrial sized $3 D$ application involving coupled poroelastic and internal acoustic domains.
\end{abstract}




\section{INTRODUCTION}

In order to limit the high computational burden associated with the evaluation of multivariate Finite Element (FE) solutions, an approach based on a Reduced-Order-Model (ROM), derived after a univariate moment-matching method, is proposed. This univariate method, namely the Well-Conditioned Asymptotic Waveform Evaluation (WCAWE) method, proposed by Slone et al. [1], is shown to allow for a robust generation of basis components with good convergence properties of the associated reduced systems [1-3]. It relies on a sequence of successive derivatives of the system of interest, leading to multiple Right-Hand-Side (RHS) problems to be solved sequentially, with intermediate orthonormalization steps and correction terms ensuring the robustness of the procedure and the good conditioning of the resulting transformation matrix. This univariate basis generation procedure may be extended to a multivariate procedure. However, the cost associated with the orthonormalization and correction steps, may lead to a computationally prohibitive cost, particularly as the dimension of the variable space increases [3]. Additionally, not all cross-derivative terms may contribute significantly to the convergence of the ROM, and it may be critical to be able to reduce them to a minimum. In this contribution, an effort is made to bring the WCAWE to multivariate problems, starting with a bivariate illustration, and attempting to limit the transformation bases to a minimum in view of higher dimensionality applications. The foundation for a multi-interval strategy is also presented in view of the reconstruction of wide parametric ranges. The approach is shown to benefit from the superior robustness of the WCAWE compared to the so-called component-wise Padé approximants [3-6], previously used to derive the Nested Padé approximant method [7, 8].

In the following Section, reminders are made about the WCAWE, the details of its procedure, and the proposed extension to multivariate problems. Then, the basis for a multi-interval strategy relying on error estimation following contours of convergence intervals is introduced. Finally, the accuracy and potential of the method are presented on a 3D poro-acoustic problem.

\section{The WCAWE, a moment-matching, projection-based ROM}

\subsection{Generic multivariate problem and associated transformation}

The starting point of the WCAWE-based parametric sweep is given by a linear system of the following form,

$$
\mathbf{Z}(\mathbf{x}) \mathbf{U}(\mathbf{x})=\mathbf{F}(\mathbf{x})
$$

where $\mathrm{x}$ may be a vector of $\mathrm{N}_{x}$ independent variables corresponding to the parametric problem of interest, e.g. including the angular frequency $\omega$, material parameters, ... For the original univariate case [1], the vector reduces to a scalar such that $\mathbf{x}=[x]$. In an FE problem, $\mathbf{Z}(\mathbf{x})$, $\mathbf{U}(\mathbf{x})$, and $\mathbf{F}(\mathbf{x})$ respectively represent the system matrix of the discretized problem, the solution vector and the vector of externally applied loads.

The projection-based approach, allowing to solve for a reduced set of equations associated with Eq. (1), relies on the construction of a transformation basis emerging from successive derivatives of the solution vector in Eq. (1) at a specific point $\mathrm{x}_{0}$ in the parameter space. The WCAWE algorithm provides a robust generation of this sequence of successive derivatives, overcoming the inherently ill-conditioned transformation matrix emerging from the direct approach used in the component-wise Padé approximants [3, 4], also used for the generation of Nested Padé approximants [7, 8]. The resulting well-conditioned transformation matrix $\mathbf{V}_{\mathrm{N}}$, consisting of $\mathrm{N}$ orthonormalized basis vectors, allows for a robust, non-stagnating convergence upon increasing the size of the subspace spanned. The associated approximation of the solution 
in Eq. (1), to the order $\mathrm{N}$, is such that

$$
\widehat{\mathrm{U}}_{\mathrm{N}}(\mathbf{x})=\mathbf{V}_{\mathrm{N}} \boldsymbol{\alpha}(\mathbf{x}) \approx \mathbf{U}(\mathbf{x}),
$$

$\boldsymbol{\alpha}$ being a vector of $\mathrm{N}$ generalized coordinates. This transformation leads to a reduced system corresponding to Eq. (1), solving for the generalized coordinates in $\boldsymbol{\alpha}(\mathbf{x})$,

$$
\mathbf{V}_{\mathrm{N}}^{T} \mathbf{Z}(\mathbf{x}) \mathbf{V}_{\mathrm{N}} \boldsymbol{\alpha}(\mathbf{x})=\mathbf{V}_{\mathrm{N}}^{T} \mathbf{F}(\mathbf{x})
$$

\subsection{A multivariate WCAWE basis generation procedure}

A generic expression of the multivariate WCAWE algorithm, as proposed in this contribution, may be given in $\mathbf{x}=\mathbf{x}_{0}$ by the following multiple right-hand-side procedure,

$$
\left\{\begin{array}{c}
\mathbf{Z}^{(0)} \overline{\mathbf{v}}_{1}=\mathbf{F}^{(0)} \\
\quad \text { Normalization } \overline{\mathbf{v}}_{1} \longrightarrow \mathbf{v}_{1} \\
\mathbf{Z}^{(0)} \overline{\mathbf{v}}_{2}=\mathbf{F}^{(1)} \mathbf{e}_{1}^{T} \mathbf{P}_{\mathbf{Q}_{\mathbf{1}}}(2,1) \mathbf{e}_{1}-\mathbf{Z}^{(1)} \mathbf{v}_{1} \\
\text { Othonormalization } \overline{\mathbf{v}}_{2} \longrightarrow \mathbf{v}_{2} \\
\vdots \\
\mathbf{Z}^{(0)} \overline{\mathbf{v}}_{n}=\left(\sum_{j=1}^{(n-1)}\left(\mathbf{F}^{(n)} \mathbf{e}_{1}^{T} \mathbf{P}_{\mathbf{Q}_{\mathbf{1}}}(n, j) \mathbf{e}_{n-j}\right)-\mathbf{Z}^{(1)} \mathbf{v}_{n-1}\right. \\
\left.\quad-\sum_{j=2}^{(n-1)}\left(\mathbf{Z}^{(j)} \mathbf{v}_{n-j} \mathbf{P}_{\mathbf{Q}_{\mathbf{2}}}(n, j) \mathbf{e}_{n-j}\right)\right) \\
\text { Othonormalization } \overline{\mathbf{v}}_{n} \longrightarrow \mathbf{v}_{n} \\
\vdots \quad\left(\sum_{j=1}^{(\mathrm{N}-1)}\left(\mathbf{F}^{(\mathrm{N})} \mathbf{e}_{1}^{T} \mathbf{P}_{\mathbf{Q}_{\mathbf{1}}}(\mathrm{N}, j) \mathbf{e}_{\mathbf{N}-j}\right)-\mathbf{Z}^{(1)} \mathbf{v}_{\mathbf{N}-1}\right. \\
\mathbf{Z}^{(0)} \overline{\mathbf{v}}_{\mathbf{N}}= \\
\left.\quad-\sum_{j=2}^{(\mathrm{N}-1)}\left(\mathbf{Z}^{(j)} \mathbf{v}_{\mathbf{N}-j} \mathbf{P}_{\mathbf{Q}_{\mathbf{2}}}(\mathbf{N}, j) \mathbf{e}_{\mathbf{N}-j}\right)\right) \\
\text { Othonormalization } \overline{\mathbf{v}}_{\mathbf{N}} \longrightarrow \mathbf{v}_{\mathbf{N}}
\end{array}\right.
$$

where a modified Gram-Schmidt orthonormalization step is performed between each vector generation by the multiple RHS systems in Eq. (4), and where

- $\mathbf{Z}^{(k)}$ stands for the differentiation of the system matrix $\mathbf{Z}$, in $\mathbf{x}=\mathbf{x}_{0}$, to the "cumulative" order $k$, regardless of the distribution of the differentiation between the independent variables $x_{i}$. In other words, given the notation $\partial_{x_{j}}^{i_{j}}(\cdot)$ for the partial derivative with respect to $x_{j}$ at order $i_{j}$, and the convention $\partial_{x_{j}}^{0}(\cdot)=(\cdot)$, then $\mathbf{Z}^{(k)}$ results from the partial derivative orders summation such that

$$
\mathbf{Z}^{\left(\sum_{j=1}^{\mathrm{N}_{x}} i_{j}\right)}=\left(\prod_{j=1}^{\mathrm{N}_{x}} \partial_{x_{j}}^{i_{j}}\right) \mathbf{Z},
$$

with the aforementioned convention implying that $\mathbf{Z}^{(0)}=\mathbf{Z}$. 
- $\mathbf{e}_{k}$ is a unitary standard basis vector associated with the $k^{\text {th }}$ component of the solution vector,

- $\overline{\mathbf{v}}_{k}$ is the non-orthonormalized vector generated in the $k^{\text {th }}$ iteration of the procedure,

- $\mathbf{v}_{k}$ is the basis vector orthonormalized against $\mathbf{v}_{k-1}$, generated after the $k^{\text {th }}$ iteration of the procedure,

- $\mathbf{P}_{\mathbf{Q}_{\omega}}(\alpha, \beta), \omega=1,2$, corresponds to the RHS correction terms, chosen to be associated with the modified Gram-Schmidt orthonormalisation process [1].

The orthonormalized and non-orthonormalized bases, $\mathbf{V}_{\mathrm{N}}$ and $\overline{\mathbf{V}}_{\mathrm{N}}$ respectively, are related by

$$
\mathrm{V}_{\mathrm{N}}=\overline{\mathbf{V}}_{\mathrm{N}} \mathrm{Q}^{-1}
$$

where $\mathrm{Q}$ is an $\mathrm{N} \times \mathrm{N}$ upper triangular, nonsingular matrix containing the modified GramSchmidt coefficients. More precisely, column $k$ of $\mathbf{Q}$ contains the successive coefficients resulting from the projection of partially orthonormalized $\overline{\mathbf{v}}_{k}$ on the orthnormalized vectors $\mathbf{v}_{j}, j<k$, and $Q_{k k}$ corresponds to the norm of $\mathbf{v}_{k}$ before its normalization. The correction terms $\mathbf{P}_{\mathbf{Q}_{\omega}}(\alpha, \beta), \omega=1,2$, are given by the following product of block matrices extracted from Q,

$$
\mathbf{P}_{\mathbf{Q}_{\omega}}(\alpha, \beta)=\prod_{t=\omega}^{\beta} \mathbf{Q}_{[t: \alpha-\beta+t-1, t: \alpha-\beta+t-1]}^{-1}
$$

Further discussions on the choice of the RHS correction coefficients other than associated with the Gram-Schmidt coefficients, may be found in Ref. [1].

The approximated solution is evaluated at all DOFs from the generalized coordinates vector $\boldsymbol{\alpha}$, after Eq. (2).

Adapting the WCAWE algorithm from univariate problems to multivariate ones lies in the generation of sequences of RHS vectors, emerging from sequences of iteratively differentiated matrices $\mathbf{Z}^{(k)}$. The choice adopted here is to generate such sequences independently, thus generating a set of $\mathrm{N}_{\mathrm{V}}$ bases $\left\{\mathrm{V}_{\mathrm{N}}^{1}, \cdots, \mathrm{V}_{\mathrm{N}}^{\mathrm{N}_{\mathrm{V}}}\right\}$ associated with $\mathrm{N}_{\mathrm{V}}$ sequences of iteratively differentiated matrices $\mathbf{Z}^{(k)}$. These bases, in principle all of the same size $\mathbf{N}$, each consist of orthonormalized basis vectors, but these are naturally not mutually orthonormalized from one to the next basis. These are not even guaranteed to produce linearly independent subsets of vectors upon merging these bases. A simple way to ensure this, as well as to reduce the basis to a minimum number of basis vectors is to proceed to a compression, or a component selection, via a Singular Value Decomposition (SVD) of the merged set of bases. Either way, the initial step consists in an SVD of the merged basis after concatenation, $\mathbf{V}_{\mathrm{N}}^{\mathrm{mer}}=\left[\mathbf{V}_{\mathrm{N}}^{1} \cdots \mathbf{V}_{\mathrm{N}}^{\mathrm{N}_{\mathrm{v}}}\right]$, resulting in

$$
\mathbf{V}_{\mathrm{N}}^{\mathrm{mer}}=\sum_{i=1}^{\mathrm{N}_{\mathrm{mer}}} \sigma_{i} \mathbf{w}_{i}^{1} \mathbf{w}_{i}^{\mathrm{r} T}
$$

where $\mathrm{N}_{\text {mer }}$ corresponds to the total number of basis vectors in $\mathbf{V}_{\mathrm{N}}^{\mathrm{mer}}$, i.e. $\mathrm{N}_{\text {mer }}=\mathrm{N} \cdot \mathrm{N}_{\mathrm{V}} ; \sigma_{i}, \mathbf{w}_{i}^{1}$, and $\mathbf{w}_{i}^{\mathrm{r}}$ correspond to the singular values, left and right singular vectors respectively.

From the SVD, either the (left) singular vectors associated with the highest singular values are selected as the components of the reduced merged basis, or the compression of the merged basis associated with the highest singular values is performed from the selected left singular vectors, right singular vectors and their corresponding singular values. Here the former approach 


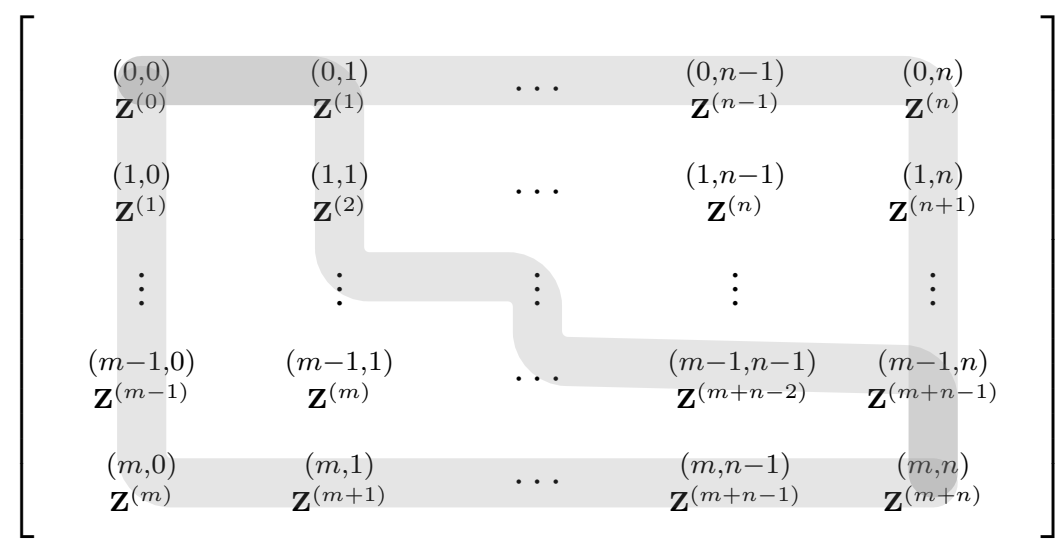

Figure 1: Illustration of three possible bivariate derivation paths for the system matrix $\mathbf{Z}$ up to the "cumulated order" $(m+n)$.

is used, such that assuming a descending-ordered sequence of singular values $\left(\sigma_{1} \cdots \sigma_{\mathrm{N}_{\text {mer }}}\right)$, the reduced, merged basis $\mathbf{V}^{*}$ is

$$
\mathbf{V}^{*}=\left\{\mathbf{w}_{i}^{1} \mid i \in\left(1, \mathrm{~N}_{\text {mer }}\right) \wedge \sigma_{i} \geq \sigma_{\text {thresh }}\right\}
$$

where $\sigma_{\text {thresh }}$ corresponds to the empirically chosen threshold value for the selection of the singular values resulting from the decomposition in Eq. (8).

The resulting reduced basis $\mathbf{V}^{*}$, consisting of $\mathrm{N}_{\mathrm{V}^{*}}$ vectors, is then used in place of the univariate transformations corresponding to Eq. (2) and Eq. (3), in order to solve a reduced set of equations at most involving $\mathrm{N}_{\mathrm{mer}}$ DOFs.

\subsection{Sequences of multivariate differentiation}

The sequential WCAWE procedure above depends on the generation of sequences of differentiated system matrices to successive "cumulated multivariate orders" $k$, i.e. $\mathbf{Z}^{(k)}$. The choice of these sequences is addressed empirically in this contribution, and illustrated for the case of bivariate problems. For the bivariate case, the cumulated differentiation orders and the associated differentiation paths may be represented in a matrix form, see Fig. 1 .

The matrix sequences obtained from these differentiation paths of the system matrix subsequently feed the procedure presented in Eq. (4).

The rationale behind choosing a limited number of derivation paths is connected to the potential inefficiency resulting from the attempt to calculate all multivariate derivatives for a given set of maximum orders. Preliminary tests based on the methodology introduced in the previous section rapidly confirm the emergence of quasi-linearly dependent basis vectors associated with different differentiation orders from distinct sequences. In practice, it is estimated that a number of sequences of the order of the dimensionality of the multivariate problem may be sufficient in order to provide good convergence properties of the ROM. For instance, 2 sequences may be sufficient for a bivariate problem. Note that this observation addresses in part the issue of dimensionality and the associated efficiency of the approach: the number of successive derivatives necessary grows linearly with the dimensionality as opposed to an exponential growth if all cross-derivatives are calculated in a fully multivariate approach. 


\section{Multi-interval, multivariate WCAWE approach}

In practice, the size of the reduction basis may be limited due to the increase of the computational cost of the procedure in Eq. (4) [3]. This implies that there may be a tradeoff between increasing the order of approximation, reflected in the size of the basis for a given reference point, and a multi-interval strategy. In order to proceed with an approximation based on multiple intervals, an error estimator is first necessary.

\subsection{Error estimation}

A Cauchy-type of convergence test is chosen in order to estimate the accuracy of the approximation without having to calculate the full solution. Provided an approximated output (scalar) quantity of interest, to the order $\mathrm{N}$, in point $\mathrm{x}$, of the form

$$
\widehat{S}_{\mathrm{N}}(\mathbf{x})=10 \log \left(\frac{\widehat{U}_{\mathrm{N}}(\mathbf{x})^{2}}{U_{\text {ref }}(\mathbf{x})^{2}}\right),
$$

the convergence is estimated in a given point $\mathbf{x}$ with the Cauchy convergence test associated with the relative error $\epsilon^{(\mathrm{N})}(\mathbf{x})=\widehat{S}_{(\mathrm{N}+2)}(\mathbf{x})-\widehat{S}_{(\mathrm{N})}(\mathbf{x})$, such that

$$
\epsilon^{(\mathrm{N})}(\mathbf{x}) \leq \epsilon_{\max }, \quad \forall \mathbf{x} \in\left[\mathbf{x}_{\min }, \mathbf{x}_{\max }\right]
$$

Note that the convergence test involves the solutions associated with several orders of difference. Two orders of difference is suggested here for examples associated with bivariate problems; more may be required for higher dimensionality. Despite a slight reduction of the convergence range achieved, a sufficiently enriched basis reduces the possibility of an erroneous estimate to occur, and leads to a conservative measure of the actual approximation error.

A Cauchy-type of convergence test however requires an evaluation of two solutions in each point of interest. It is consequently necessary to limit the number of evaluations of the relative error for each interval in order to limit the extra cost associated with the error estimation.

\subsection{Contour-following and multi-interval strategy}

In order to reduce the number of points at which the error estimation needs to be made, a contour-following strategy is implemented. Starting from the reference point for each interval, an isocontour is determined (Moore-Neighbour Tracing algorithm), associated with the max error $\epsilon_{\max }$ in Eq. (11). This contour subsequently allows to fit a regular shape (e.g. a rectangle for a bivariate problem) providing a simplified measure of the interval of convergence. The successive dimensions of the intervals of convergence may further be used in order to anticipate the choice of subsequent reference points for approximation of the solution on neighbour intervals.

There are several simple strategies possible in order to fill an entire parametric solution space with smaller intervals of convergence. One may iteratively choose reference points in direct connection with the range of convergence of the previously calculated intervals, as adopted for instance in Ref. [8]. Another possibility is to successively choose the reference points such that they are the furthest away from any boundary of the parametric space or the converged contours.

\section{Application: poro-acoustic parametric sweep}

The proposed multivariate approach is tested on an average-sized problem consisting of the interior cavity of a passenger train, treated with a $15-\mathrm{cm}$ layer of sound absorbing porous material on the top surface. A time-harmonic point source is defined at a corner of the cavity, and 


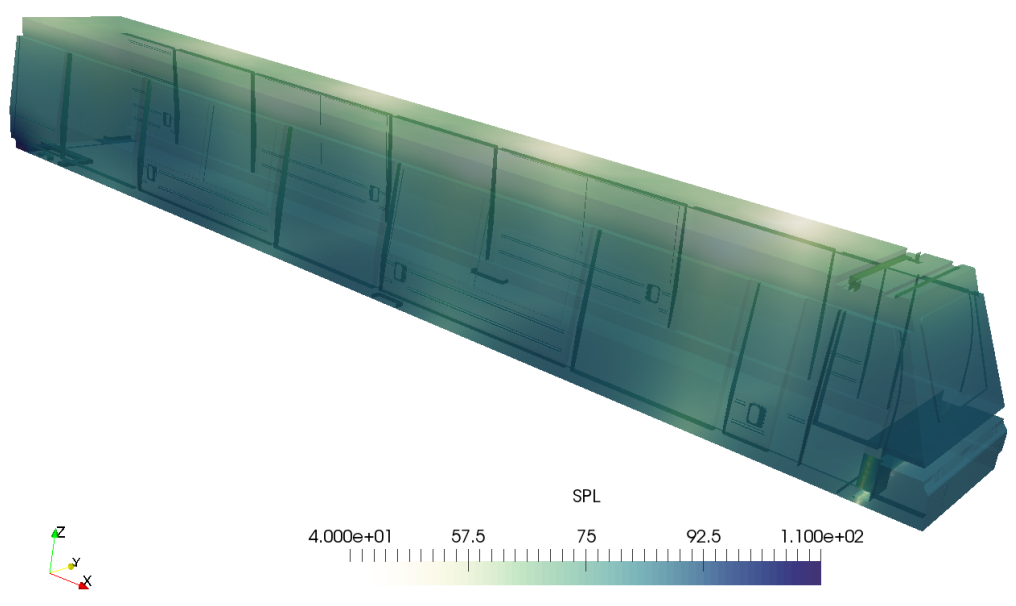

Figure 2: Sound pressure level of the damped solution at $63 \mathrm{~Hz}, \Phi=25000$ N.s.m ${ }^{-4}$, scale $40-110 \mathrm{~dB}$.

parametric sweeps with respect to both the frequency and the static airflow resistivity of the porous layer are performed. All boundary walls are considered as rigid walls, except from the porous boundary. The porous boundary is modelled by an equivalent fluid formulation, consisting of a modified Helmholtz equation where the equivalent speed of sound is complex and frequency-dependent, given by

$$
\tilde{c_{\mathrm{p}}}=\frac{c_{0}}{\sqrt{\frac{1-i \Phi}{\rho_{0} \omega}}}
$$

where $(\tilde{\cdot})$ denotes a complex-valued quantity, $\rho_{0}=1.21 \mathrm{~kg} \cdot \mathrm{m}^{-3}$ is the ambient density of the air saturating the pores and $c_{0}=343 \mathrm{~m} . \mathrm{s}^{-1}$ the speed of sound in the air. $\omega=2 \pi f$ is the angular frequency associated with the time-harmonic excitation, and $\Phi$ is the static flow resistivity, associated with the viscous dissipation in the porous material.

After a standard expression of the problem in its weak form and subsequent discretization by a Galerkin method, the finite element problem has the general form

$$
\left(\mathbf{K}_{\mathbf{a}}-\frac{\omega^{2}}{c_{0}^{2}} \mathbf{M}_{\mathbf{a}}+\mathbf{K}_{\mathbf{p}}-\frac{\omega^{2}}{{\tilde{c_{\mathrm{p}}}}^{2}} \mathbf{M}_{\mathbf{p}}\right) \tilde{\mathbf{U}}=\mathbf{F}
$$

where $\square_{\mathbf{a}}$ correspond to air cavity global matrices, and $\square_{\mathbf{p}}$ to porous global matrices. $\tilde{\mathrm{U}}$ is the vector of nodal unknowns (acoustic pressure fluctuation here). The right-hand-side vector $\mathbf{F}$, associated with the time-harmonic acoustic excitation is in practice only non-zero at a few DOFs. The FE problem in Eq. (13) is evidently of the form of the generic problem in Eq. (1), thus suitable for the multivariate approach proposed. The discretized problem consists of around 300000 acoustic pressure DOFs.

Fig. 2 illustrates the solution at a given point of the parametric sweep, for a frequency of $63 \mathrm{~Hz}$, and a flow resistivity of 25000 N.s.m ${ }^{-4}$.

The reference solution for the bivariate parametric sweep, where $f \in[50,150] \mathrm{Hz}$ and $\Phi \in$ $[3000,50000]$ N.s.m ${ }^{-4}$, at a point in the cavity, is plotted in Fig. 3. 


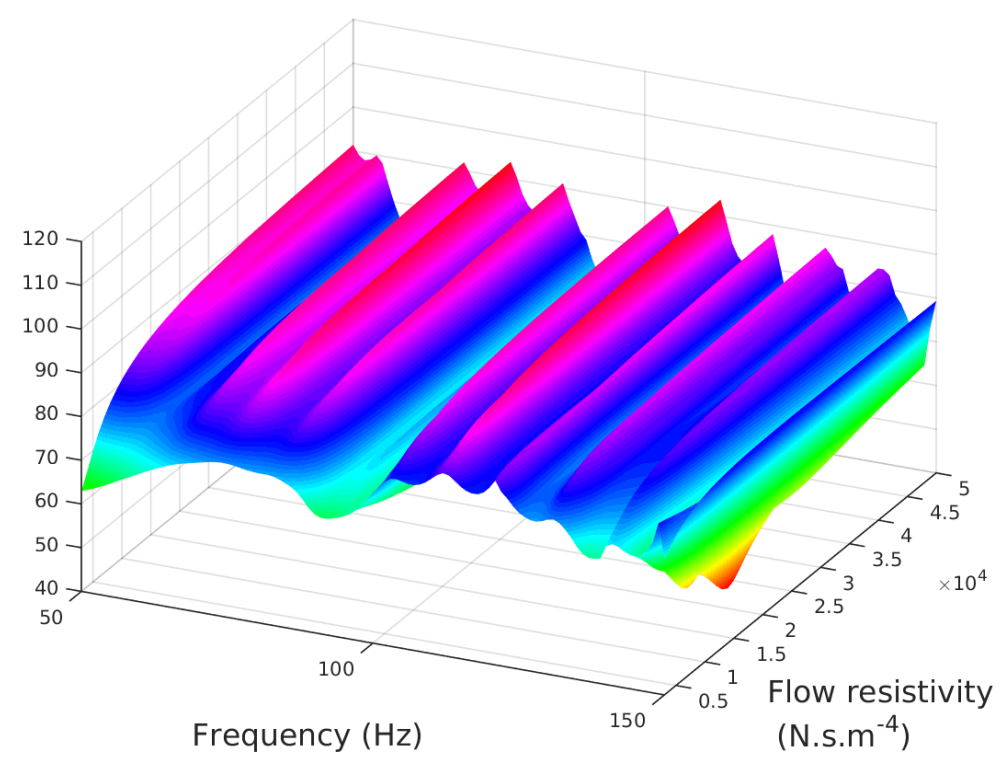

Figure 3: Reference solution. Sound pressure level at a point in the acoustic cavity, parametric solution, in $\mathrm{dB}$.

The multivariate WCAWE procedure proposed here is applied for an approximated solution based on one reference point in the parametric space for the construction of the transformation basis.

Two configurations are tested regarding the number of sequences necessary in order to produce the reduced merged basis $\mathbf{V}^{*}$ of Eq. (9). The first configuration includes 3 sequences: the 2 "outer" sequences, and one "diagonal" sequence, as presented in Fig. 1. Given that in this configuration, some components added by the third sequence are not selected by the SVD, and that the increased size of the reduced basis $\mathbf{V}^{*}$ does not noticeably improve the accuracy of the approximation, the second configuration includes only the two "outer" sequences. The singular value threshold $\sigma_{\text {thresh }}$, used for the selection of the main components for the reduced basis $\mathbf{V}^{*}$, is chosen to be such that $\sigma_{\text {thresh }}=\sigma_{\max } \cdot 10^{-15}$, where $\sigma_{\max }$ corresponds to the largest singular value in the decomposition of Eq. (8).

In Fig. 4, the convergence of the approximated bivariate solution is illustrated for three different orders of approximation, for a reduced basis $\mathrm{V}^{*}$ based on the two "outer" sequences.

Given the smooth variation of the solution with respect to the static flow resistivity, it is natural that a lower order of approximation is required than for the frequency. In this illustration, the order of differentiation for the static flow resistivity is set to 9. Three increasing orders of differentiation are plotted for the frequency, from order 19 to order 55. The degree of accuracy reached for the latter case, although using one point only at $f=88 \mathrm{~Hz}$ and $\Phi=25000$ N.s.m ${ }^{-4}$ for the construction of the basis, highlights the potential of the method.

Table 1 provides a brief comparison of the size of the bases obtained from the proposed procedure when 2 or 3 sequences of differentiation are used prior to the SVD. A combination of the facts that some vectors added by the 3rd sequence are filtered out by the SVD, while all vectors emerging from the combination of the 2 "outer" sequences are kept, indicates that a 2 -sequence approach may be sufficient for the bivariate case. In particular, no improvement of 


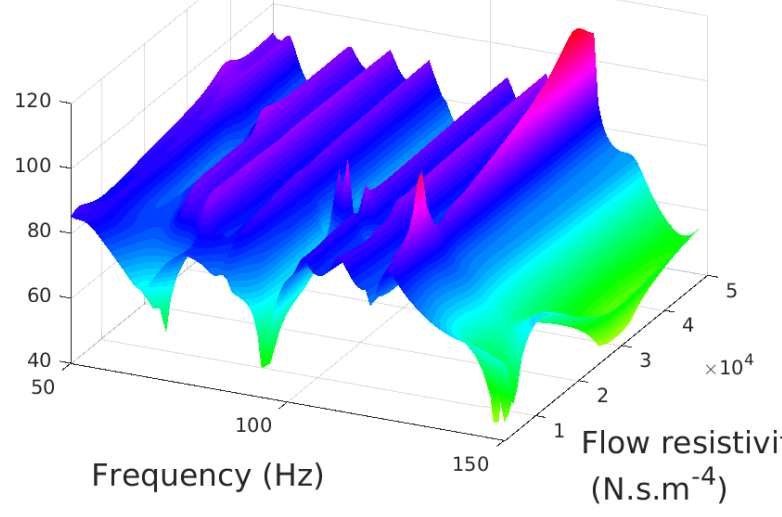

(a)

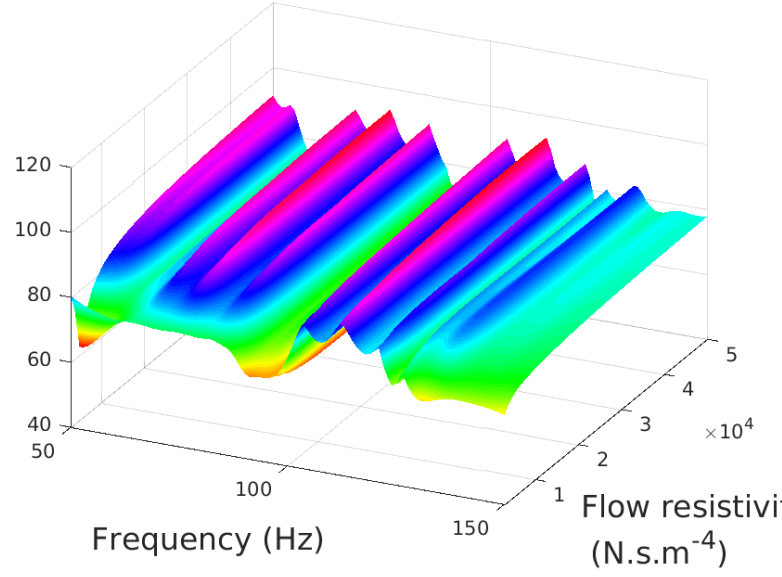

(c)

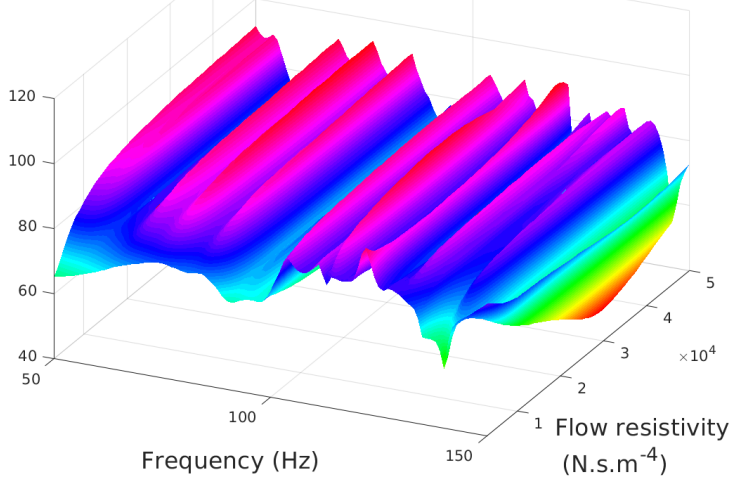

(e)

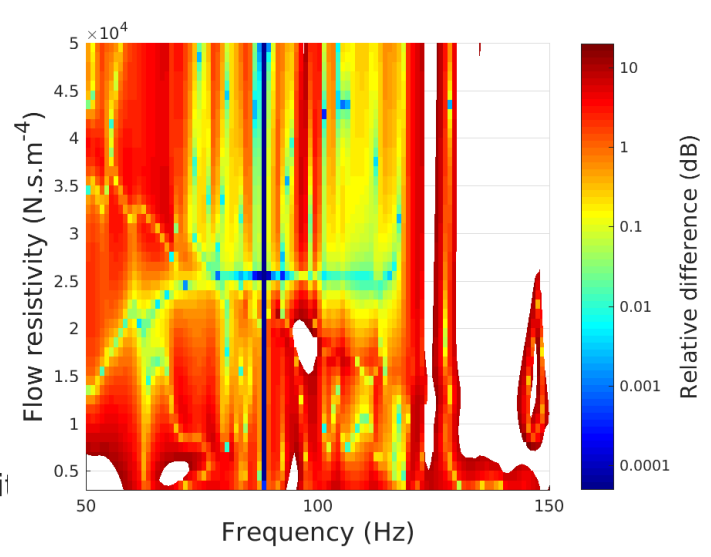

(b)

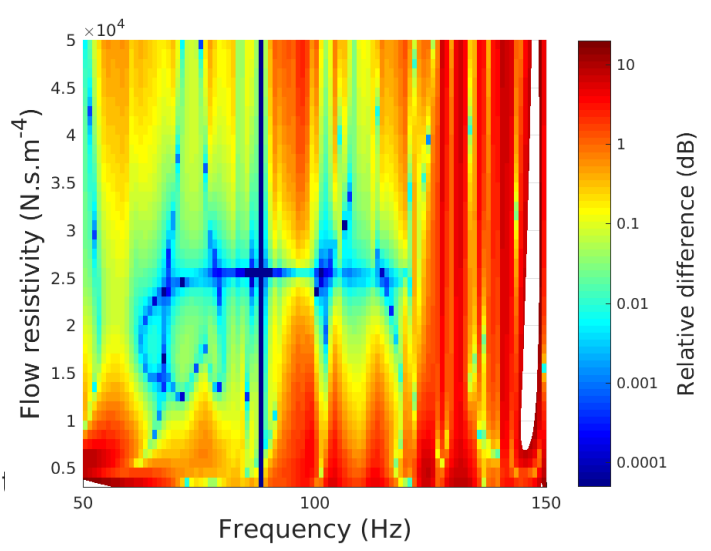

(d)

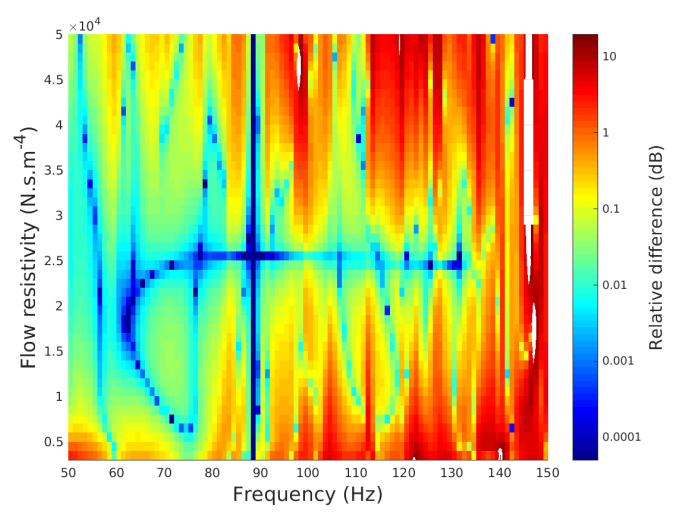

(f)

Figure 4: Convergence of the one-point WCAWE-approximated solution. SPL at a single point, in $\mathrm{dB}$. Reference parametric point for the WCAWE basis procedure: $f=88 \mathrm{~Hz}$ and $\Phi=25000$ N.s.m ${ }^{-4}$. (a) (b) Derivative orders 19/9 for frequency/resistivity; (c) (d) Derivative orders 31/9 for frequency/resistivity; (e) (f) Derivative orders 55/9 for frequency/resistivity. Approximation (left) and relative difference to the reference solution (right). 


\begin{tabular}{c||ccc} 
& $\mathrm{N} \mathrm{vec/basis}$ & $\mathrm{N}_{\text {mer }}$ & $\mathrm{N}_{\mathrm{V}^{*}}$ \\
\hline \hline 2-sequence & $29(9+19+1)$ & $56(29+27)$ & 56 \\
3-sequence & $29(9+19+1)$ & $83(29+27+27)$ & 79 \\
\hline 2-sequence & $41(9+31+1)$ & $80(41+39)$ & 80 \\
3-sequence & $41(9+31+1)$ & $119(41+39+39)$ & 112 \\
\hline 2-sequence & $65(9+55+1)$ & $128(65+63)$ & 128
\end{tabular}

Table 1: Overview of the size of the reduced basis $\mathbf{V}^{*}$ emerging from the 2- and 3-sequence procedure associated with the results in Fig, 4

convergence and accuracy was observed by adding the third sequence, thus indicating a minor contribution from the basis vectors emerging from this additional sequence. This also reflects a conservative threshold value for $\sigma_{\text {thresh. }}$. Finally, the fact that no basis vector is filtered out by the SVD, when all vectors emerging from the combination of the 2 "outer" sequences are kept, implies that the SVD is in this case not associated with a selection of the basis components, but rather with a re-orthogonalization of the reduced basis $\mathrm{V}^{*}$.

Limiting the expansion to derivative orders 20/12 for the frequency and flow resistivity, respectively, Fig. 5illustrates the contour-following, filling and shape-fitting approach in order to define sub-intervals of convergence. The error estimation is calculated along the contours only, in Figs. 5a and 5c, assuming a monotonous increase of the relative error inside the contours around the reference point of expansion, and a tolerance of $\epsilon_{\max }=0.02 \mathrm{~dB}$. These contours may subsequently be fitted, as plotted in grey in Figs. $5 \mathrm{~b}$ and $5 \mathrm{~d}$, with a regular shape connected to the shape of the parametric space, i.e. a rectangle for this bivariate example.

A first attempt for a multi-interval approximation of the full bivariate parametric space is plotted in Figs. 6. This approach is based on a surface-fitting matching the surface area of the estimated contour, augmented by an overestimation factor, here set to 1.5. This over-estimation factor is justified by the slow increase of the error beyond the convergence contours, provided that the error tolerance is low enough (here $0.02 \mathrm{~dB}$ ). The first reference point, associated with the first interval in Fig. 6a, is chosen following a preliminary step testing the convergence range associated with a reference point in the center of the parametric space. Then, subsequent intervals are chosen based on the dimensions of the last 2 calculated intervals, in the closest neighbourhood possible to the last calculated interval. When the parametric space has been entirely covered, the algorithm attempts to bridge the largest gaps, based on the dimension of the smallest interval of convergence. Fig. 6b shows in white the estimated range of convergence after 3 intervals (starting from the low frequency and low flow resistivity ranges). Fig. 6c shows the estimated range of convergence after 9 intervals, after which the entire parametric range is "covered". Finally, Fig. 6d plots the estimated range of convergence after the largest gaps have been added extra reference points, including 16 intervals in total. This implies the calculation of 16 multiple RHS problems consisting of 33 RHS vectors in order to build the complete set of reduced bases, to be contrasted with the full solution to be calculated at $101 \times 48=4848$ points for the reference solution.

The proposed approach is currently being tested for its robustness with respect to a wide range of problems, starting, as done here, with bi-parametric problems. 


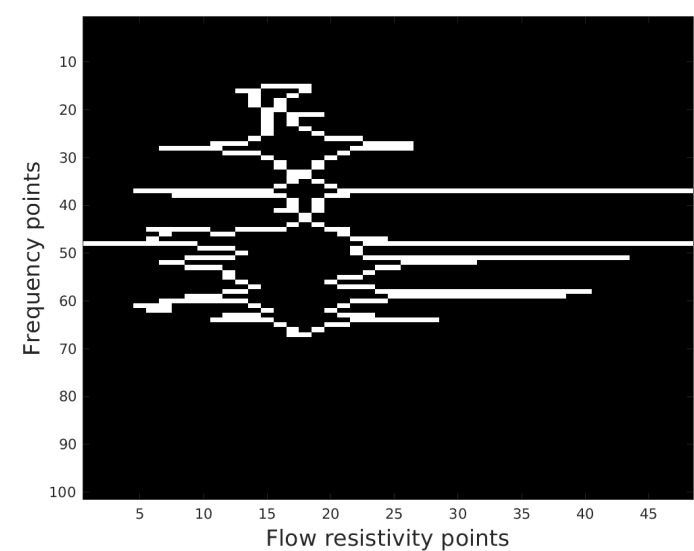

(a)

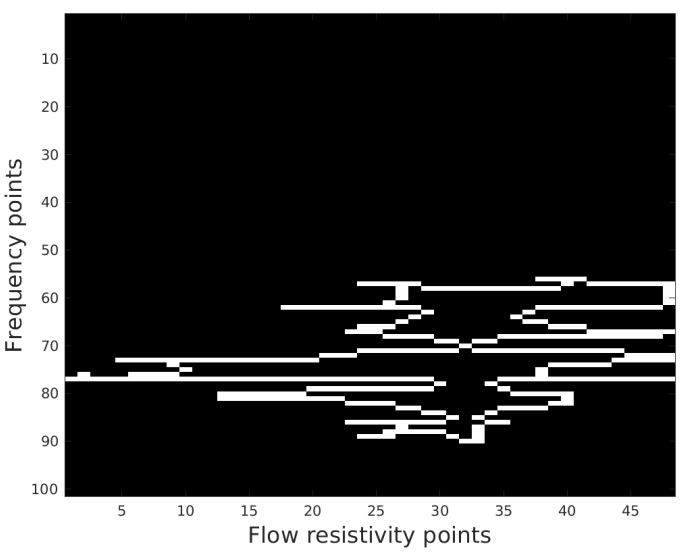

(c)

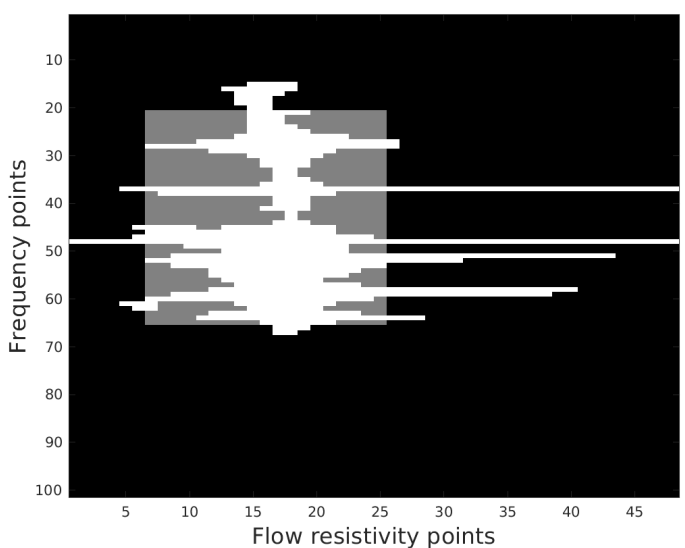

(b)

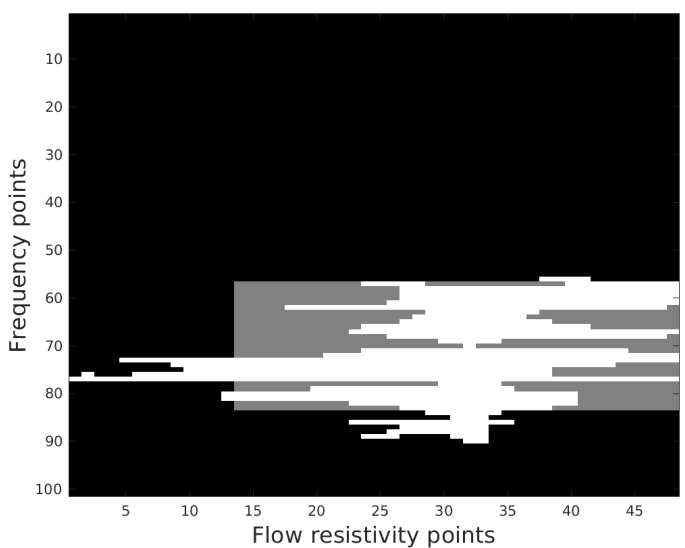

(d)

Figure 5: Illustration of the contour-following and shape fitting strategy for a single interval associated with the poro-acoustic bivariate problem. (a) and (c) Isocontour following of the max relative error; (b) and (d) Isocontour filling and fitting with a regular shape (rectangle).

\section{CONCLUSIONS}

In this contribution, a multivariate approach based on the Well-Conditioned Asymptotic Waveform Evaluation algorithm is proposed. It relies on the generation of basis vectors emerging from sequences of incrementally differentiated system matrices at parametric points of expansion. These sequences, efficiently generated by a multiple right-hand-side type of problem, are subsequently merged and further reduced via a singular value decomposition.

The convergence of the approach is illustrated on a bivariate poro-acoustic application of about 300000 degrees of freedom. The limitation to a reduced set of sequences is promising in the scope of using the proposed approach for higher dimensionality. An extension to a multiinterval approximation strategy, based on the efficient use of error estimator is also tested and currently under further developments in connection with its robustness. 


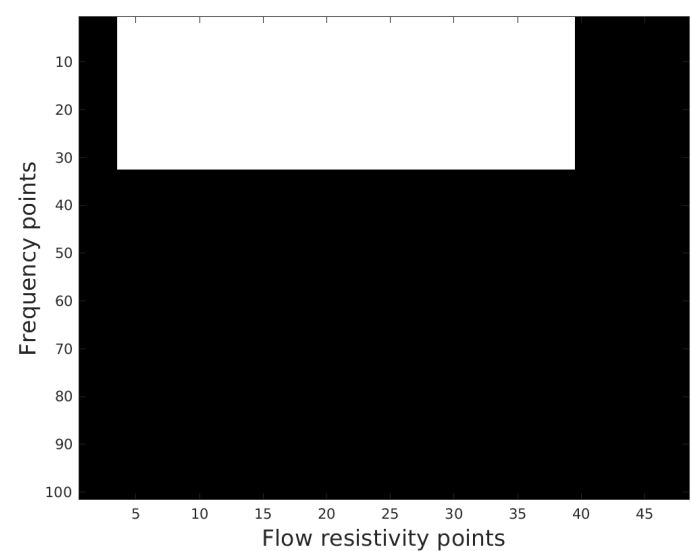

(a)

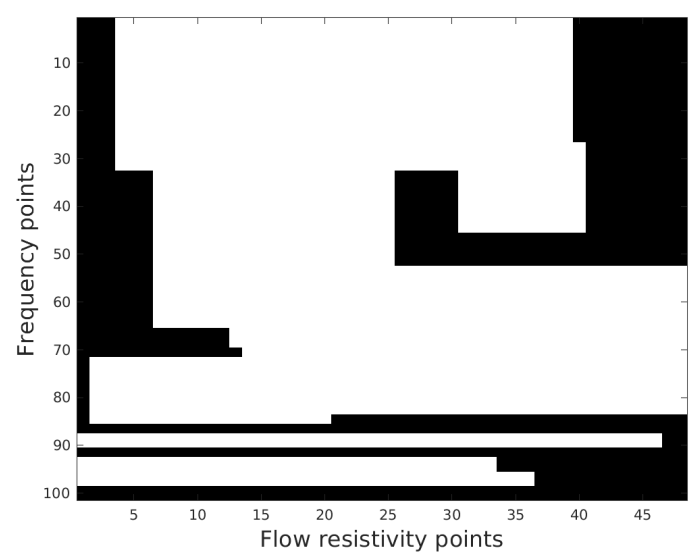

(c)

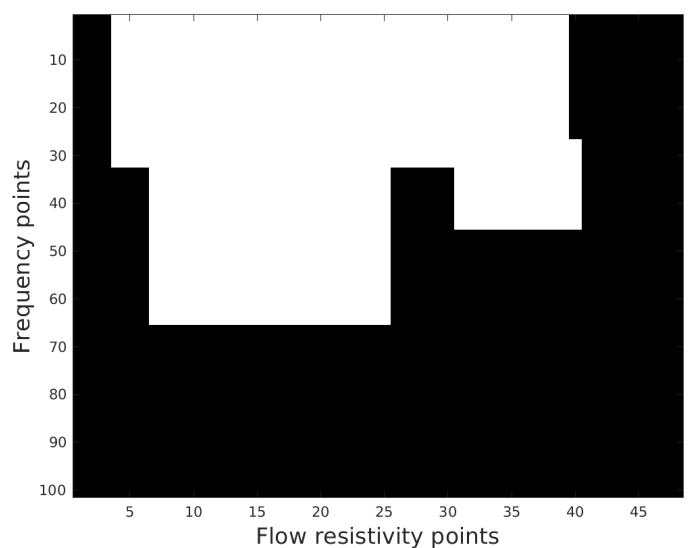

(b)

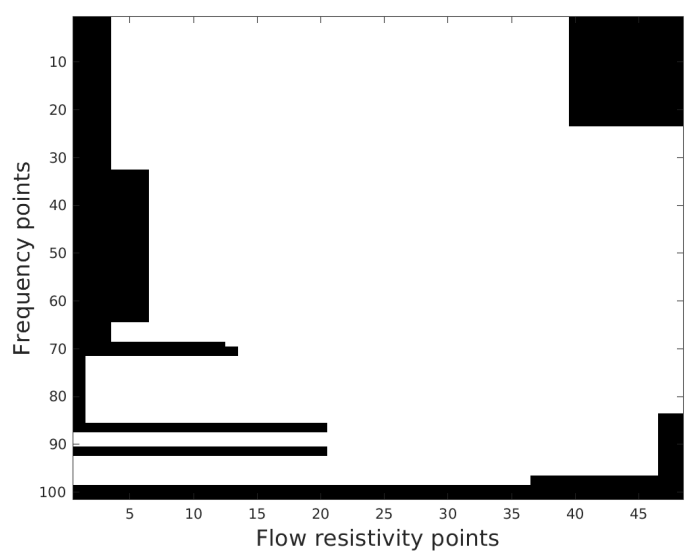

(d)

Figure 6: Convergence of a multi-interval approach for bases with fixed derivative orders 20/12, with a tolerance of $\epsilon_{\max }=0.02 \mathrm{~dB}$. (a): 1 convergence interval, (b), 3 convergence intervals, (c): 9 convergence intervals, (d): 16 convergence intervals.

\section{ACKNOWLEDGEMENTS}

The financial support from the Swedish Research Council (Vetenskapsrådet VR Grant 201504925 for the first author) and the Centre for ECO2 Vehicle Design (VINNOVA Grant 201605195) are gratefully acknowledged.

\section{REFERENCES}

[1] R. D. Slone, R. Lee, and J.-F. Lee. Well-conditioned asymptotic waveform evaluation for finite elements. IEEE Transactions on Antennas and Propagation, 51(9):2442-2447, 2003.

[2] M. S. Lenzi, S. Lefteriu, H. Beriot, and W. Desmet. A fast frequency sweep approach using Padé approximations for solving Helmholtz finite element models. Journal of Sound and Vibration, 332(8):1897-1917, 2013. 
[3] R. Rumpler, and P. Göransson. An assessment of two popular Padé-based approaches for fast frequency sweeps of time-harmonic finite element problems. Proceedings of Meetings on Acoustics, 30(1):022003, 2017. DOI: 10.1121/2.0000649

[4] G.A. Baker and P.R. Graves-Morris. Padé approximants, volume 59. Cambridge University Press, 1996.

[5] R. Djellouli, C. Farhat, and R. Tezaur. A fast method for solving acoustic scattering problems in frequency bands. Journal of Computational Physics, 168(2):412-432, 2001.

[6] R. Rumpler, P. Göransson, and J.-F. Deü. A finite element approach combining a reducedorder system, padé approximants, and an adaptive frequency windowing for fast multifrequency solution of poro-acoustic problems. International Journal for Numerical Methods in Engineering, 97(10):759-784, 2014.

[7] P. Guillaume. Nested multivariate padé approximants. Journal of computational and applied mathematics, 82(1):149-158, 1997.

[8] R. Rumpler, P. Göransson, and H.J. Rice. An adaptive strategy for the bivariate solution of finite element problems using multivariate nested Padé approximants. International Journal for Numerical Methods in Engineering, 100(9):689-710, 2014.

[9] R. Rumpler. Padé approximants and the modal connection: towards increased robustness for fast parametric sweeps. International Journal for Numerical Methods in Engineering, 113(1):65-81, 2018. 